\title{
Fabrication of a 3.5-GHz Solidly Mounted Resonator by Using an AlScN Piezoelectric Thin Film
}

\author{
Chan-Yu Chung ${ }^{1}$, Ying-Chung Chen ${ }^{1}$, Yu-Cheng Chen ${ }^{1}$, Kuo-Sheng Kao ${ }^{2, *}$ and Yu-Chen Chang ${ }^{1}$ \\ 1 Department of Electrical Engineering, National Sun Yat-sen University, Kaohsiung 80424, Taiwan; \\ d063010001@student.nsysu.edu.tw (C.-Y.C.); ycc@mail.ee.nsysu.edu.tw (Y.-C.C.); \\ m063010121@student.nsysu.edu.tw (Y.-C.C.); d043010004@student.nsysu.edu.tw (Y.-C.C.) \\ 2 Department of Computer and Communication, SHU-TE University, Kaohsiung 82445, Taiwan \\ * Correspondence: kks@stu.edu.tw
}

Citation: Chung, C.-Y.; Chen, Y.-C.; Chen, Y.-C.; Kao, K.-S.; Chang, Y.-C. Fabrication of a 3.5-GHz Solidly Mounted Resonator by Using an AlScN Piezoelectric Thin Film. Coatings 2021, 11, 1151. https:// doi.org/10.3390/coatings11101151

Academic Editor: Roman A. Surmenev

Received: 16 July 2021

Accepted: 18 September 2021

Published: 23 September 2021

Publisher's Note: MDPI stays neutral with regard to jurisdictional claims in published maps and institutional affiliations.

Copyright: (c) 2021 by the authors. Licensee MDPI, Basel, Switzerland. This article is an open access article distributed under the terms and conditions of the Creative Commons Attribution (CC BY) license (https:// creativecommons.org/licenses/by/ $4.0 /)$.

\begin{abstract}
In this study, a 3.5-GHz solidly mounted resonator (SMR) was developed by doping scandium in aluminum nitride to form $\mathrm{AlScN}$ as the piezoelectric thin film. Molybdenum (Mo) of $449 \mathrm{~nm}$ thickness and silicon dioxide $\left(\mathrm{SiO}_{2}\right)$ of $371 \mathrm{~nm}$ thickness were used as the high and low acoustic impedance films, respectively, which were alternately stacked on a silicon substrate to form a Bragg reflector. Then, an alloy target with atomic ratio of $15 \% \mathrm{Sc}$ was adopted to deposit the piezoelectric AlScN thin film on the Bragg reflector, using a radio frequency magnetron sputtering system. The characteristics of the c-axis orientation of the $\mathrm{AlScN}$ thin films were optimized by adjusting sputtering parameters as sputtering power of $250 \mathrm{~W}$, sputtering pressure of $20 \mathrm{mTorr}$, nitrogen gas ratio of $20 \%$, and substrate temperature of $300{ }^{\circ} \mathrm{C}$. Finally, a metal top electrode was coated to form a resonator. The X-ray diffraction (XRD) analysis showed that the diffraction peak angles of the AlScN film shifted towards lower angles in each crystal phase, compared to those of AlN film. The energy dispersive X-ray spectrometer (EDX) analysis showed that the percentage of scandium atom in the film is about $4.5 \%$, regardless of the sputtering conditions. The fabricated resonator exhibited a resonance frequency of $3.46 \mathrm{GHz}$, which was a small deviation from the preset resonance frequency of $3.5 \mathrm{GHz}$. The insertion loss of $-10.92 \mathrm{~dB}$ and the electromechanical coupling coefficient of $2.24 \%$ were obtained. As compared to the AlN-based device, the AlScN-based resonator exhibited an improved electromechanical coupling coefficient by about two times.
\end{abstract}

Keywords: SMR; AlScN; 3.5-GHz; BAW

\section{Introduction}

With continuous technological advancement, the rapid development of RF microelectromechanical devices is urgently required for manufacturing wireless communication equipment. The miniaturization and multi-functionality of the equipment have completely changed the way people communicate. For example, mobile communication devices used in vehicles and aviation, global positioning system (GPS) and military satellites are all applications of wireless communication. Moreover, with the advent of fifth-generation mobile communication technology (5G), the frequency spectrum, quality and characteristics of filters are becoming more and more important in 5G or higher frequency bands.

There are many types of filters; among them, the acoustic wave filter is of the most concern. The acoustic wave filters can be divided into surface acoustic wave (SAW) filters [1] and bulk acoustic wave (BAW) filters [2,3]. Compared with surface acoustic wave filters, bulk acoustic wave filters can more easily achieve a high frequency range $(>2 \mathrm{GHz})$, and have a smaller volume, excellent device characteristics, higher power endurance and wider application frequency range, among other advantages, which make the bulk acoustic wave filters highly valued in future mobile communication applications.

A BAW resonator comprises a piezoelectric film, sandwiched between the upper and lower electrodes. To effectively limit the acoustic wave energy in the piezoelectric 
layer, at present, face-etched [4-7], back-etched [8-13], and solidly mounted structures are used to avoid the loss of sound wave energy through the substrate. Solidly mounted resonators (SMRs) are composed of high and low-acoustic-impedance materials, alternately deposited on the substrate at a quarter resonant wavelength $(\lambda / 4)$ to form a Bragg reflector. Depending on the design, the reflector can be used as a low-acoustic-impedance interface, similar to air, to prevent the sound wave energy from escaping the substrate, or as a highacoustic-impedance interface to reflect the sound wave energy back into the piezoelectric layer. As SMR suppresses or reflects sound waves in piezoelectric materials through the Bragg reflector, there is no need to etch the cavity from the substrate when manufacturing the device, which results in a stable structure and high yield [14-22].

Aluminum nitride $(\mathrm{AlN})$ and zinc oxide $(\mathrm{ZnO})$ are popular piezoelectric materials used in BAW devices. $\mathrm{ZnO}$ has a high electromechanical coupling coefficient $k_{t}{ }^{2}$, but a low longitudinal acoustic wave velocity. AlN has a high longitudinal wave velocity and is therefore preferred for realizing sub-6 GHz components in 5G systems. Both AlN and $\mathrm{ZnO}$ are excellent piezoelectric materials [23]. However, because of the current trend of high-frequency communications, AlN has attracted more attention than $\mathrm{ZnO}$.

In addition to the development of high-frequency components, increases in the $k_{t}{ }^{2}$ value of a material are noteworthy. Many methods have been adopted to improve the $k_{t}{ }^{2}$ value of a material. According to Tasnádi et al. [24], the hexagonal structure of ScN will contribute to the piezoelectricity of $\mathrm{Sc}_{x} \mathrm{Al}_{1-x} \mathrm{~N}(x<0.5)$ thin films. Farrer et al. discovered that $\mathrm{ScN}$ has an unstable wurtzite structure and a stable hexagonal structure, due to the isostructural phase transition of wurtzite to a layered hexagonal structure $[25,26]$. Sc-IIIA-N compounds exhibit excellent piezoelectric properties. Among the IIIA compounds, AlN exhibits the highest Curie temperature and thermal stability [27]. AlN and Sc can be combined to form $\mathrm{Sc}_{x} \mathrm{Al}_{1-x} \mathrm{~N}$ alloy films as novel piezoelectric materials for acoustic wave components.

\section{Materials and Methods}

\subsection{SMR Design}

In this study, a 3.5-GHz SMR was developed using an AlScN piezoelectric film, sandwiched by a bottom and a top electrode. A low-roughness Bragg reflector was deposited on the substrate to prevent the dissipation of acoustic wave energy. In the SMR structure, the upper part of the piezoelectric layer is an air or vacuum interface, while the lower part is a reflective Bragg reflector formed by alternately stacking high-acoustic-impedance materials and low-acoustic-impedance materials. The SMR structure can be divided into the two modes of $\lambda / 4$ mode and $\lambda / 2$ mode. In this study, an $\lambda / 2$ mode resonator is adopted, in which the thickness of each layer of the reflective Bragg reflector is $\lambda / 4$, and that of the piezoelectric layer is $\lambda / 2$, where $\lambda$ is the resonance wavelength. According to the structure of the reflective layer, whether the impedance of the interface between the piezoelectric layer and the reflective layer is low-impedance or high-impedance can be determined. Looking into the reflective layer from the piezoelectric layer, if the acoustic impedance of the 1,3, and 5 layers is lower than that of the 2, 4, and 6 layers, the overall impedance of the reflective layer tends to zero, and there is a free interface between the Bragg reflector and the piezoelectric layer, as shown in Figure 1. If the wavelength of the sound wave does not comply with a wavelength of $\lambda / 2$, the sound wave does not form a standing wave, which results in energy dissipation. Generally, the piezoelectric film is a $\lambda / 2$ type resonator, which has a larger effective electromechanical coupling coefficient than that of the $\lambda / 4$ type resonator [28].

In this study, high-acoustic-impedance material of molybdenum (Mo) and low-acousticimpedance material of silicon dioxide $\left(\mathrm{SiO}_{2}\right)$ were used as the reflective high and low impedance layers, respectively, to construct the Bragg reflector on Si substrate. The thicknesses of $\mathrm{Mo}$ and $\mathrm{SiO}_{2}$ films can be derived from the formula $v=f \times 4 d$, where $\mathrm{v}$ is the bulk acoustic wave velocity of $\mathrm{Mo}_{\text {or }} \mathrm{SiO}_{2}$ film; $f$ is the resonance center frequency; and 
$\mathrm{d}$ is the thickness of $\mathrm{Mo}$ or $\mathrm{SiO}_{2}$ film, which are calculated as approximately $449 \mathrm{~nm}$ and $371 \mathrm{~nm}$, respectively, to accommodate the $3.5 \mathrm{GHz}$ frequency response.

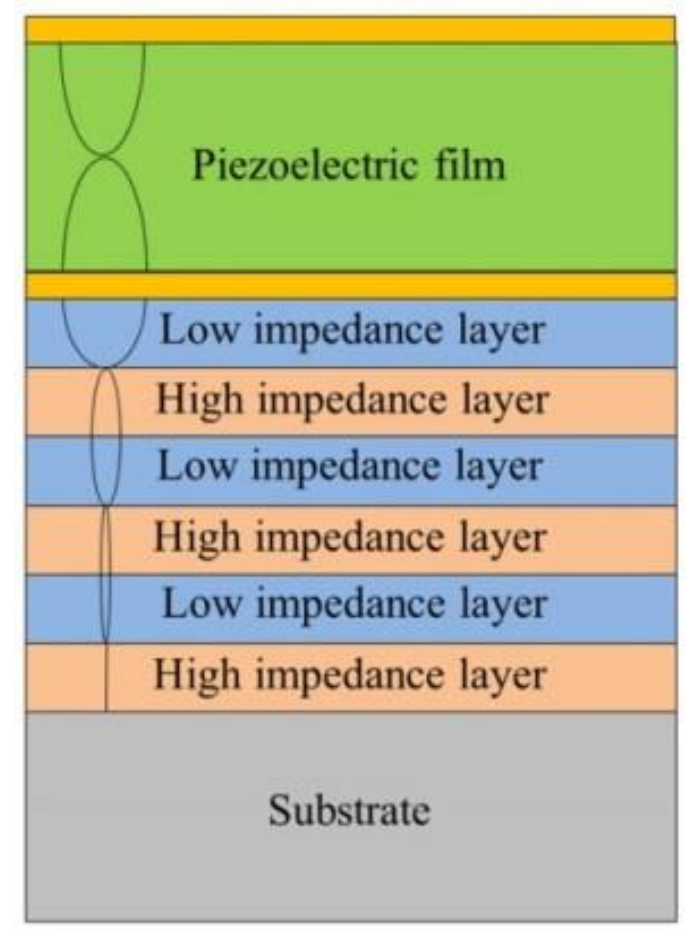

Figure 1. The schematic of a $\lambda / 2$ mode resonator.

$\mathrm{An} \mathrm{Al}(85 \%)-\mathrm{Sc}(15 \%)$ alloy target was used to deposit $\mathrm{AlScN}$ piezoelectric films, using a reactive RF magnetron sputtering system. The quality of the piezoelectric layer considerably affects the characteristics of the device. Therefore, the characteristics of the piezoelectric film were analyzed through scanning electron microscopy (SEM, JEOL-6700 Field Emission SEI/BEI type scanning electron microscope, JEOL, Ltd., Tokyo, Japan), X-ray diffraction (XRD, Bruker, Billerica, MA, USA), and energy dispersive X-ray spectroscopy (EDX, JEOL, Ltd., Tokyo, Japan) to determine the optimal sputtering parameters. SEM was used to analyze the surface and cross-sectional structures of the films under various sputtering conditions. XRD was performed using an X-ray diffractometer (Bruker D2 Phaser system, Bruker, Billerica, MA, USA) to analyze the crystalline orientation and the peak strength of X-ray diffraction of AlScN film and determine its optimal sputtering parameters.

Finally, the required thickness of the piezoelectric film was determined to fabricate SMR devices, which had their frequency responses and electromechanical coupling coefficients measured using a network analyzer E5071C (Keysight, Santa Rosa, CA, USA).

\subsection{SMR Parametric Properties}

In BAW devices, the effective electromechanical coupling coefficient $k_{t}{ }^{2}$ and the thickness of the piezoelectric layer affect the resonator bandwidth, insertion loss, and resonance frequency. Therefore, the deposition of thin film with low-acoustic wave loss and high electromechanical coupling coefficient is critical.

\subsection{Measurement of Electromechanical Coupling Coefficient $k_{t}^{2}$}

The electromechanical coupling coefficient is defined as the ability to convert mechanical energy to electrical energy and vice versa. The greater the electromechanical coupling coefficient, the better the conversion effect. In addition, the higher the effective electromechanical coupling coefficient of the resonator constituting the filter, the greater the bandwidth of the combined filter [29]. 
The electromechanical coupling coefficient is mainly determined by two parameters, namely the parallel resonant frequency $f_{p}\left(\omega_{p}\right)$ at which the imaginary part of impedance is maximum, and the series resonant frequency $f_{s}\left(\omega_{s}\right)$ at which the imaginary part of impedance is zero. The effective electromechanical coupling coefficient $k_{t}^{2}$ is calculated as follows [30]:

$$
\left(k_{t}\right)^{2}=\left(\frac{\pi^{2}}{4}\right) \cdot\left(\frac{\omega_{s}}{\omega_{p}}\right) \cdot\left(\frac{\omega_{p}-\omega_{s}}{\omega_{p}}\right)
$$

\subsection{Fabrication Processes}

In this study, on-axis direct current (DC) sputtering system and radio-frequency (RF) magnetron sputtering system were used to alternately deposit $\mathrm{Mo}$ and $\mathrm{SiO}_{2}$ films on a p-type (100) Si substrate to form three pairs of Bragg reflectors. The $\mathrm{Mo}$ and $\mathrm{SiO}_{2}$ layers were 449- and 371-nm thick, respectively. Photolithography was used to define the bottom electrode pattern. A seed layer of Ti and the bottom electrode of Mo were deposited through DC magnetron sputtering. The overlying film of $\mathrm{AlScN}$ was then deposited above the bottom electrode by using a reactive RF magnetron sputter. Finally, another photolithography process was used to define the top electrode. The Mo/Ti top electrode was fabricated on the piezoelectric film by using a DC magnetron sputter. To realize an electrical connection with the bottom electrode, photolithography was performed again for thorough hole patterning. AlScN was etched with phosphoric acid at $90{ }^{\circ} \mathrm{C}$. Thus, a $\lambda / 2$ mode SMR was fabricated. Deposition was performed through DC magnetron sputtering and RF magnetron sputtering, and patterning was realized through photolithography by using three masks. The flowchart of the fabrication process is displayed in Figure 2. Figure 3 shows the 3D representation of the structure of a solidly mounted resonator.
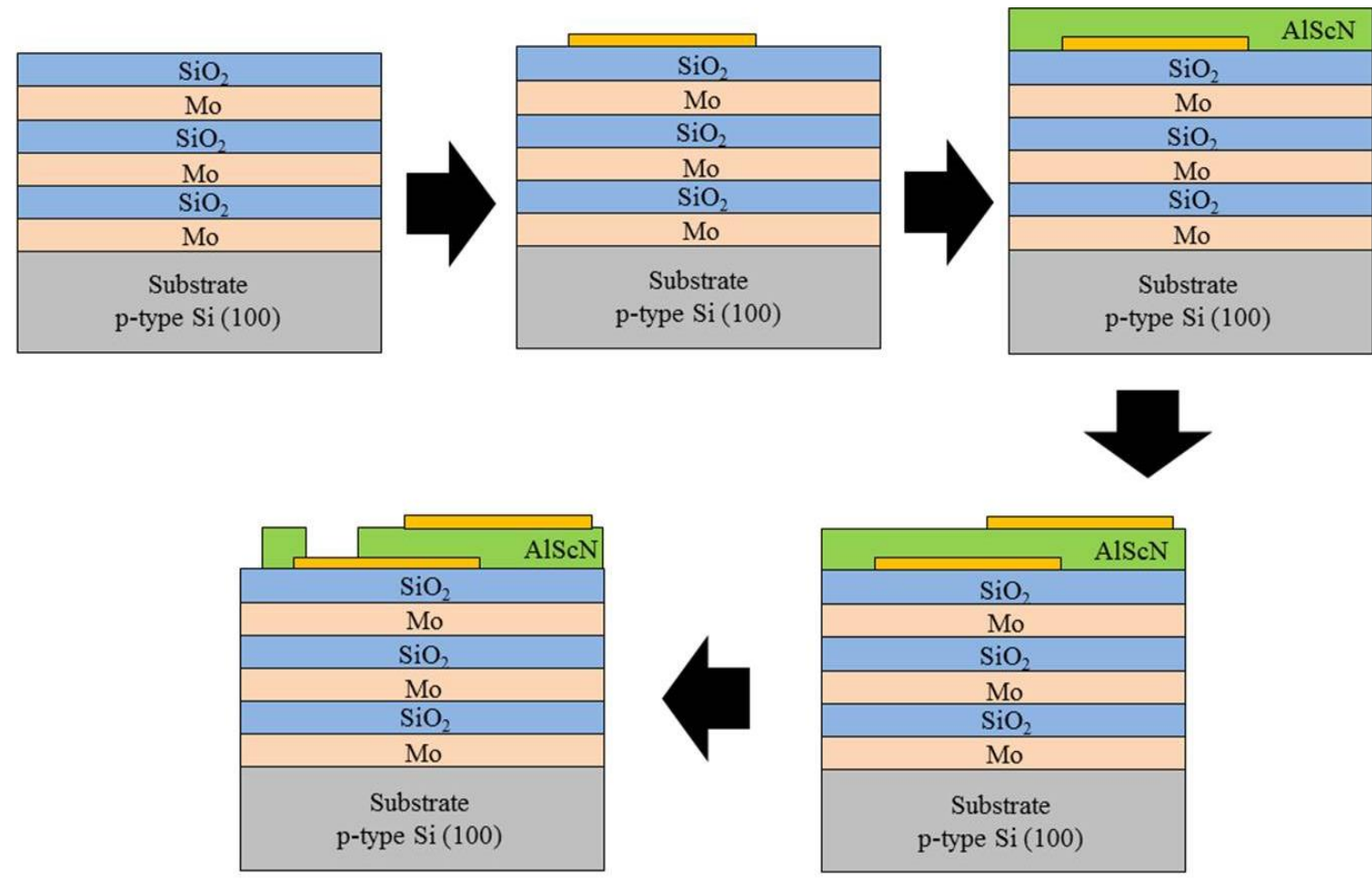

Figure 2. The flowchart of the fabrication process of the designed SMR device. 


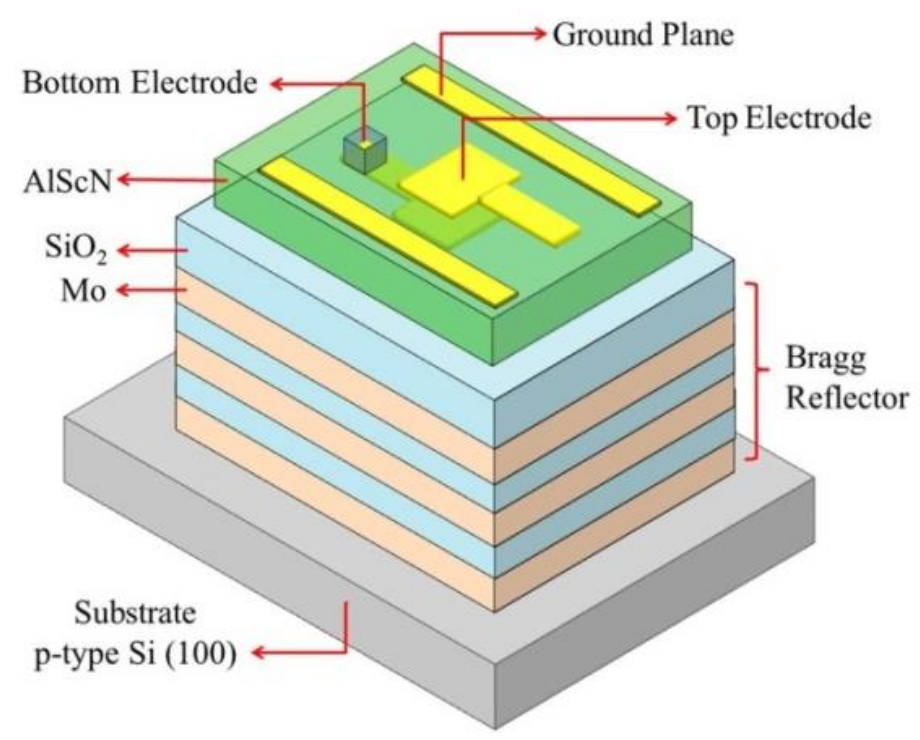

Figure 3. 3D representation of the structure of a solidly mounted resonator.

\section{Results and Discussion}

To confine acoustic energy in the piezoelectric layer and prevent acoustic scattering, the surface of the Bragg reflector should be as flat as possible [31]. The DC and RF magnetron sputters were precisely controlled to alternately deposit $\mathrm{Mo}$ and $\mathrm{SiO}_{2}$ films on a silicon substrate, to prepare a low-surface-roughness Bragg reflector. The optimal sputtering parameters of the Mo thin film were obtained using a sputtering power of $100 \mathrm{~W}$ and a sputtering pressure of 1 mTorr. Atomic force microscopy (AFM, Digital Instrument, Milano, Italy) revealed that the deposited Mo film had a surface roughness of $1.367 \mathrm{~nm}$. A sputtering power of $80 \mathrm{~W}$, sputtering pressure of $5 \mathrm{mTorr}$, and a substrate temperature of $350{ }^{\circ} \mathrm{C}$ were the optimal sputtering parameters of $\mathrm{SiO}_{2}$. AFM revealed that the $\mathrm{Mo} / \mathrm{SiO}_{2}$ structure had a surface roughness of $1.298 \mathrm{~nm}$. The conditions for a high c-axis structure of the AlScN piezoelectric film were determined by changing the sputtering parameters. In this experiment, an $\mathrm{Al}(85 \%)-\mathrm{Sc}(15 \%)$ alloy target was used to deposit the AlScN piezoelectric film with the strongest (002) crystal orientation and a highly pronounced c-axis orientation [32], using reactive RF magnetron sputtering.

SEM and XRD were performed to characterize the surfaces and cross-sectional structures, crystal characteristics, and compositions of the films. The sputtering power, sputtering pressure, and nitrogen ratio $\left(\mathrm{N}_{2} / \mathrm{N}_{2}+\mathrm{Ar}\right)$ were adjusted to deposit the AlScN films on the $\mathrm{Mo} / \mathrm{Ti} / \mathrm{Si}$ structure at a substrate temperature of $300^{\circ} \mathrm{C}$. SEM and XRD were performed on the deposited films to verify the physical properties and determine the optimal sputtering parameters.

In the experiment, the consistency of each set of samples has been verified through physical property analysis. The next set of process parameters will be executed until the reproducibility of the samples is confirmed. Firstly, the sputtering power, nitrogen ratio, and substrate temperature were fixed as $250 \mathrm{~W}, 20 \%$, and $300{ }^{\circ} \mathrm{C}$, and the sputtering pressure was adjusted to 10, 20, and 30 mTorr, respectively. Figure 4 shows the XRD diffraction patterns of the AlScN films, which shows a pronounced diffraction peak at $35.97^{\circ}$, corresponding to the (002) crystal orientation of the wurtzite structure, and that the film performs best when the sputtering pressure is $20 \mathrm{mTorr}$. There exists a diffraction peak shift phenomenon between AlScN and AlN films, the (002) crystal orientation angle of AlN is $36.079^{\circ}$ and $\mathrm{AlScN}$ is $35.97^{\circ}$, and the offset between the two is $0.109^{\circ}$. The reason for this is that $\mathrm{Sc}$ replaces $\mathrm{Al}$ atom in the lattice structure, and the change in the lattice structure causes the shift in the diffraction peak [33]. From the SEM analysis, as shown in Figure 5, the surface of the film sputtered at 20 mTorr exhibits a dense small cobblestone structure and a columnar and clear cross-section. 


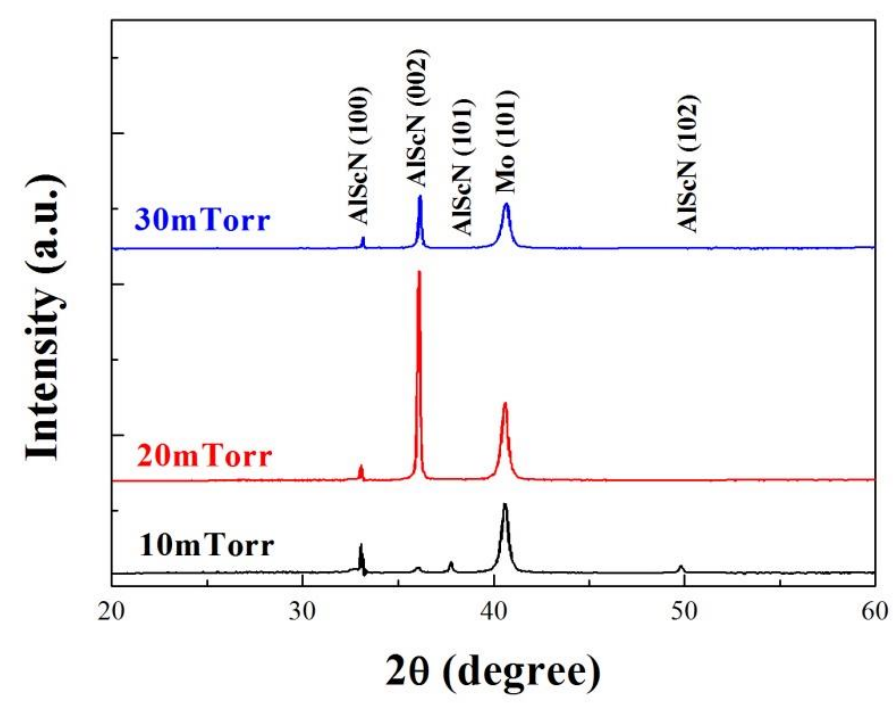

Figure 4. XRD diffraction patterns of AlScN films deposited at different sputtering pressures.
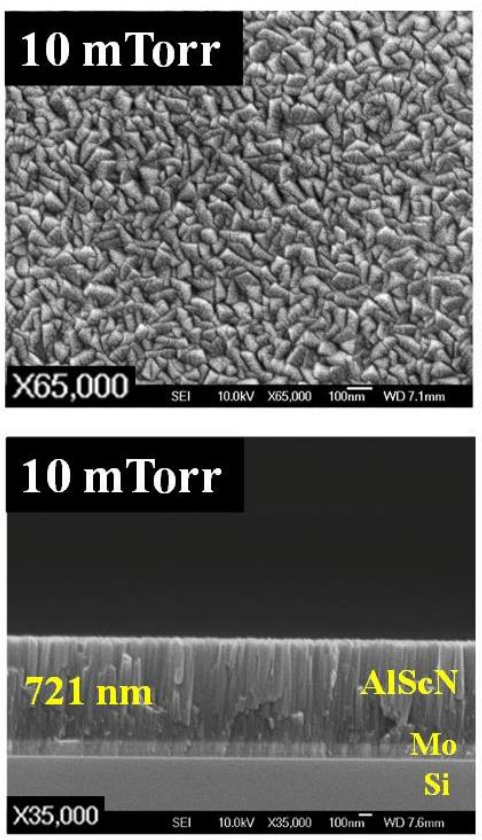

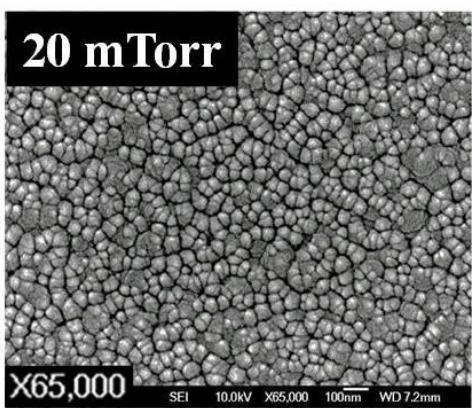

\section{0 mTorr}

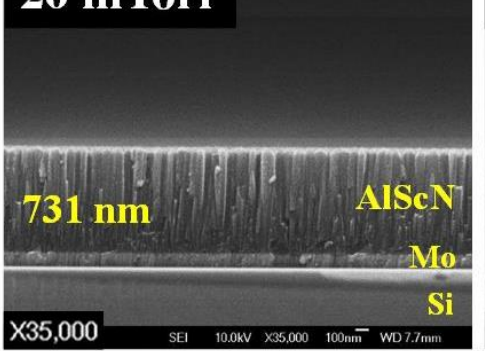

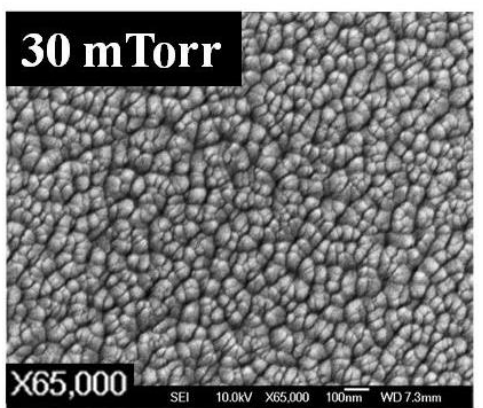

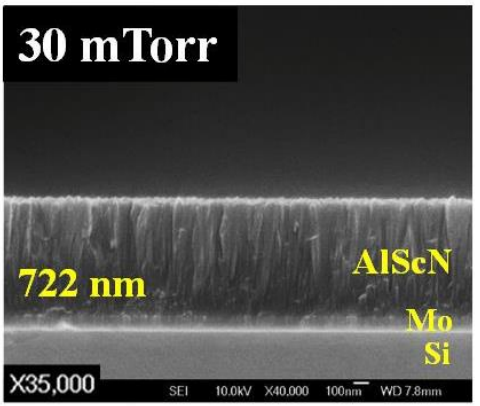

Figure 5. SEM images of the surfaces and cross-sectional structures of AlScN films deposited at different sputtering pressure.

Then, the sputtering pressure, sputtering power, and substrate temperature were fixed as $20 \mathrm{mTorr}, 250 \mathrm{~W}$, and $300{ }^{\circ} \mathrm{C}$. The nitrogen ratio was adjusted to $20 \%, 50 \%$, and $80 \%$, and the influence of the gas environment on the $\mathrm{AlScN}$ film was discussed. As shown in Figure 6, the film exhibits the strongest intensity of (002) crystal orientation at a nitrogen ratio of $20 \%$, then decreases with the increased nitrogen ratio. Figure 7 reveals that the surface of the film sputtered at the nitrogen ratio of 20 mTorr exhibits a dense cobblestone structure and an excellent columnar cross-section.

Finally, the sputtering pressure, nitrogen ratio, and substrate temperature were fixed as $20 \mathrm{mTorr}, 20 \%$, and $300{ }^{\circ} \mathrm{C}$, and the sputtering power was adjusted to 200,250 and $300 \mathrm{~W}$, respectively. As displayed in Figure 8, when the sputtering power is increased to $250 \mathrm{~W}$, the film exhibits the strongest (002) crystal orientation. Figure 9 shows the SEM images of the surfaces and cross-sectional structures of the $\mathrm{AlS} \mathrm{cN}$ films deposited under various sputtering powers. The surface of the film exhibits a dense small cobblestone structure and columnar and clear cross-section at $250 \mathrm{~W}$. The above results indicate that a chamber 
pressure of $20 \mathrm{mTorr}$, nitrogen ratio of $20 \%$, substrate temperature of $300{ }^{\circ} \mathrm{C}$, and sputtering power of $250 \mathrm{~W}$ are the optimal parameters to deposit the AlScN films in this study.

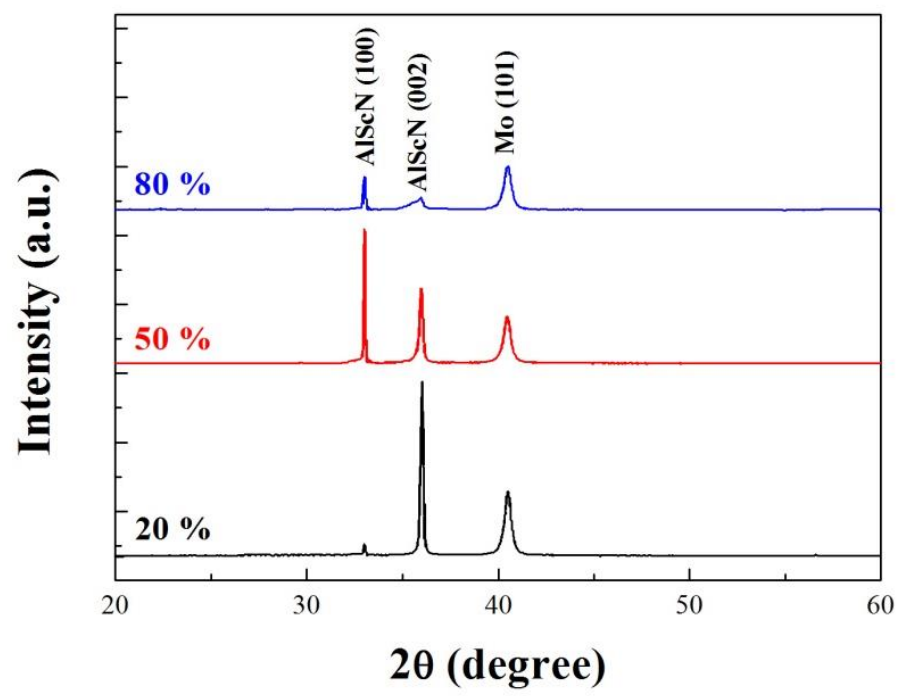

Figure 6. XRD diffraction patterns of $\mathrm{AlScN}$ films deposited at different sputtering nitrogen ratios $\left(\mathrm{N}_{2} / \mathrm{N}_{2}+\mathrm{Ar}\right)$.
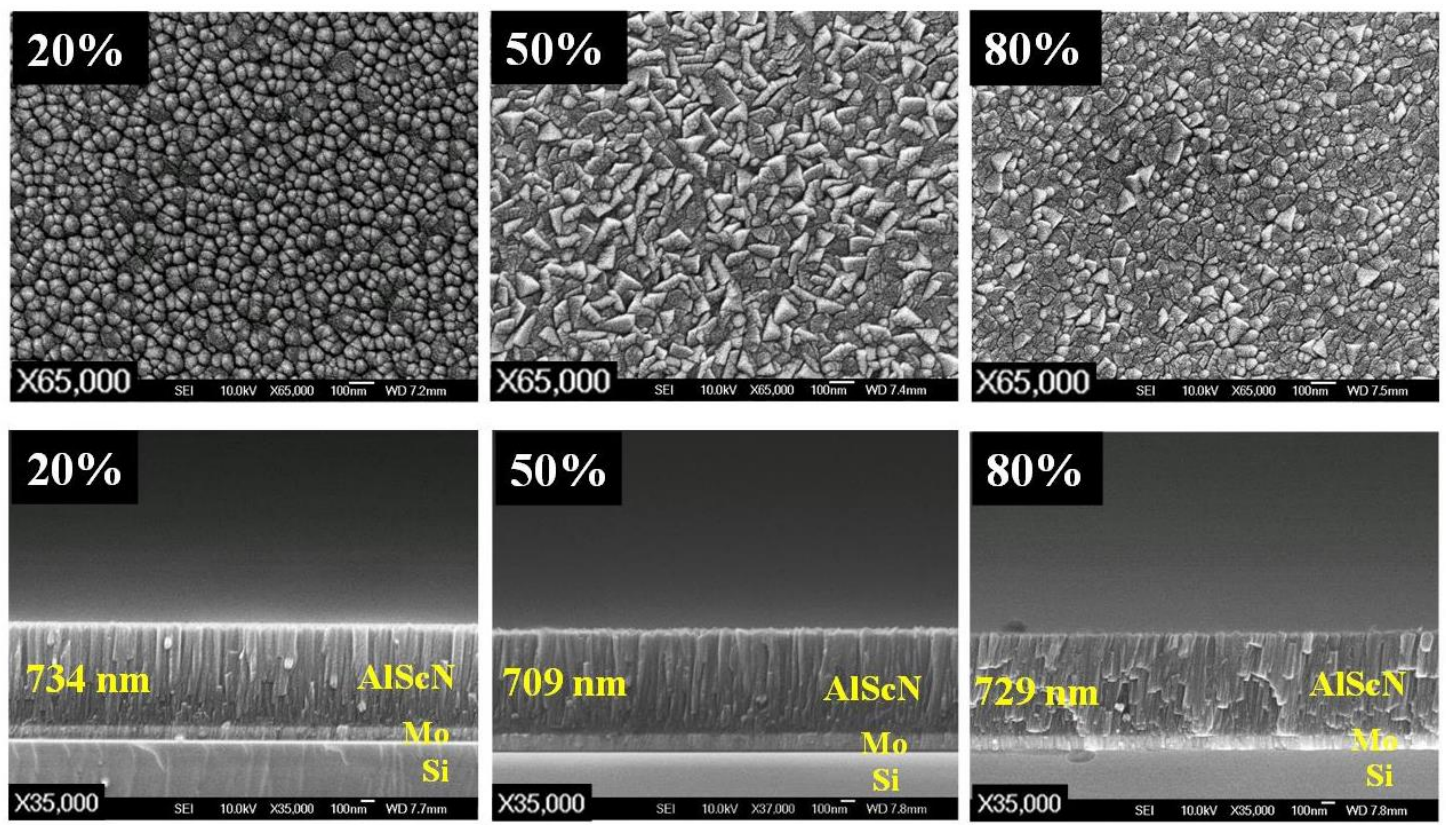

Figure 7. SEM images of the surfaces and cross-sectional structures of deposited AlScN films at different nitrogen ratio $\left(\mathrm{N}_{2} / \mathrm{N}_{2}+\mathrm{Ar}\right)$.

It is worth noting that the XRD diffraction peak angles of the AlScN films are shifted towards lower angles with $2 \theta$ of about $0.1 \sim 0.25^{\circ}$ in each crystal phase, compared to those of AlN films. The reason for this is that the larger scandium atoms replace part of the aluminum atoms in the aluminum-nitrogen bond. The crystal lattice structure of the film changes, which, in turn, causes the angle of the diffraction peak to shift.

On the other hand, the compositions of the AIScN films under different sputtering conditions were analyzed by EDS. The results show that the scandium content in the film under different sputtering pressures, nitrogen ratios, and sputtering powers is not much different, because the experiment uses an aluminum-scandium alloy target (15\% scandium) as the sputtering target. The proportion of aluminum and scandium in the target is fixed, so 
it is difficult to vary the scandium content in the film by changing the sputtering conditions. The overall results show that the percentage of scandium atom in the film is about $4.5 \%$.

In this study, Bragg reflectors with three pairs of $\mathrm{Mo} / \mathrm{SiO}_{2}$ are used in the SMR devices. In order to clarify the effect of Sc doping in AlN piezoelectric film on the characteristics of acoustic wave devices, a $3.5 \mathrm{GHz}$ SMR device with an AlN piezoelectric film was fabricated. Figure 10a shows the $\mathrm{S}_{21}$ response of the AlN-based SMR.

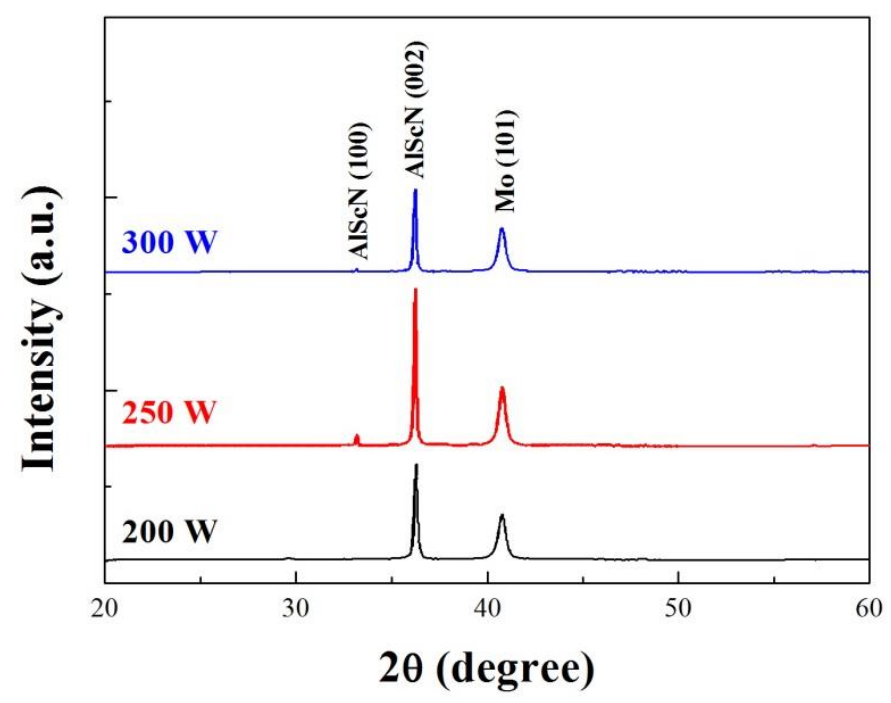

Figure 8. XRD diffraction patterns of AlScN films deposited at different sputtering power.
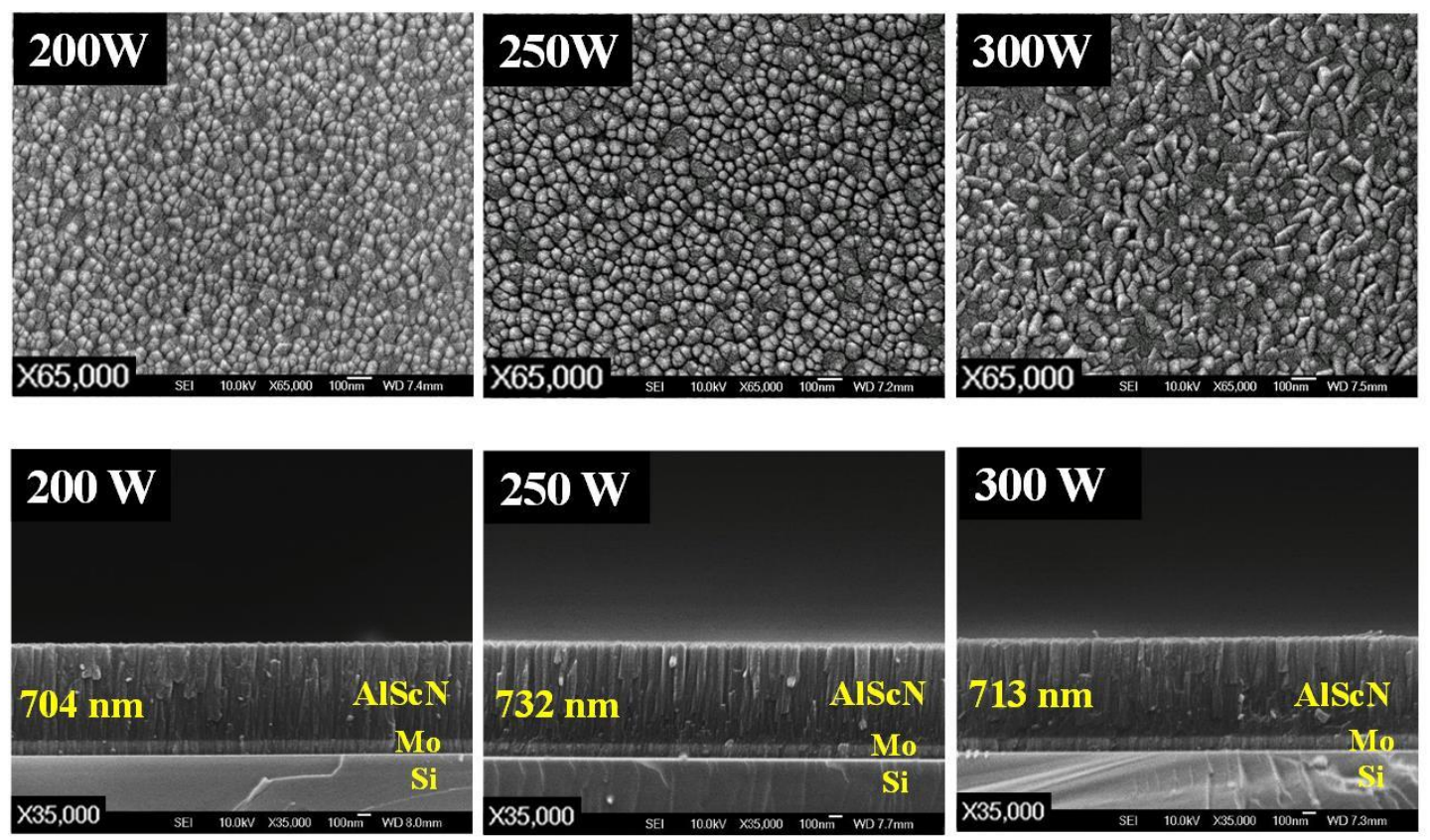

Figure 9. SEM images of the surfaces and cross-sectional structures of AlScN films deposited at different sputtering power.

In order to fabricate the designed SMR device, the thickness of the piezoelectric AlScN thin film was adjusted according to the $\lambda / 2$ mode SMR device design. In the design, based on previous experience, it is estimated that the sound velocity of the AlScN film is about $6000 \mathrm{~m} / \mathrm{s}$. Therefore, the thickness of the piezoelectric layer is preliminarily set as $863 \mathrm{~nm}$, according to the formula $v=f \times 2 d$, in which, $\mathrm{v}$ is the bulk acoustic wave velocity of AlScN film, $\mathrm{f}$ is the resonance center frequency, and $\mathrm{d}$ is the thickness of AlScN film. The film thickness and characteristics of AlScN were then taken into the simulation software. 
The simulated frequency response of $S_{21}$ is shown in Figure 10b, in which the resonance center frequency of $3.5 \mathrm{GHz}$, the electromechanical coupling coefficient $k_{t}^{2}$ of $5.45 \%$, the quality factor $\mathrm{Q}$ of 208 , and the insertion loss IL of $-10.83 \mathrm{~dB}$ are obtained. However, as the component was fabricated using an AlScN piezoelectric film of $863 \mathrm{~nm}$ thickness, a resonance response with a frequency of $2.97 \mathrm{GHz}$ is obtained. The deviation between simulated and experimental may indicate that the elastic constant of the piezoelectric film is changed due to the doping of Sc, resulting in a decrease in sound velocity compared to that estimated. Therefore, the required piezoelectric layer thickness must be adjusted to $731 \mathrm{~nm}$, derived from the calculated true sound velocity of the AlScN film of about $5126 \mathrm{~m} / \mathrm{s}$. Figure 10c shows the frequency response of $S_{21}$ of the SMR device after adjusting the thickness of the piezoelectric layer to $731 \mathrm{~nm}$, in which the electromechanical coupling coefficient $k_{t}{ }^{2}$ of $2.24 \%$, the quality factor $\mathrm{Q}$ of 183 , and the insertion loss IL of $-10.92 \mathrm{~dB}$ are obtained. Figure 11 shows the frequency response $S_{11}$ of the SMR device with the $731 \mathrm{~nm}$ thick AlScN piezoelectric film. From this, a resonance response at $3.46 \mathrm{GHz}$ can be obtained, which is near the designed resonance frequency of $3.5 \mathrm{GHz}$. It also has a return loss of $-30.62 \mathrm{~dB}$, as shown in Figure 11 .
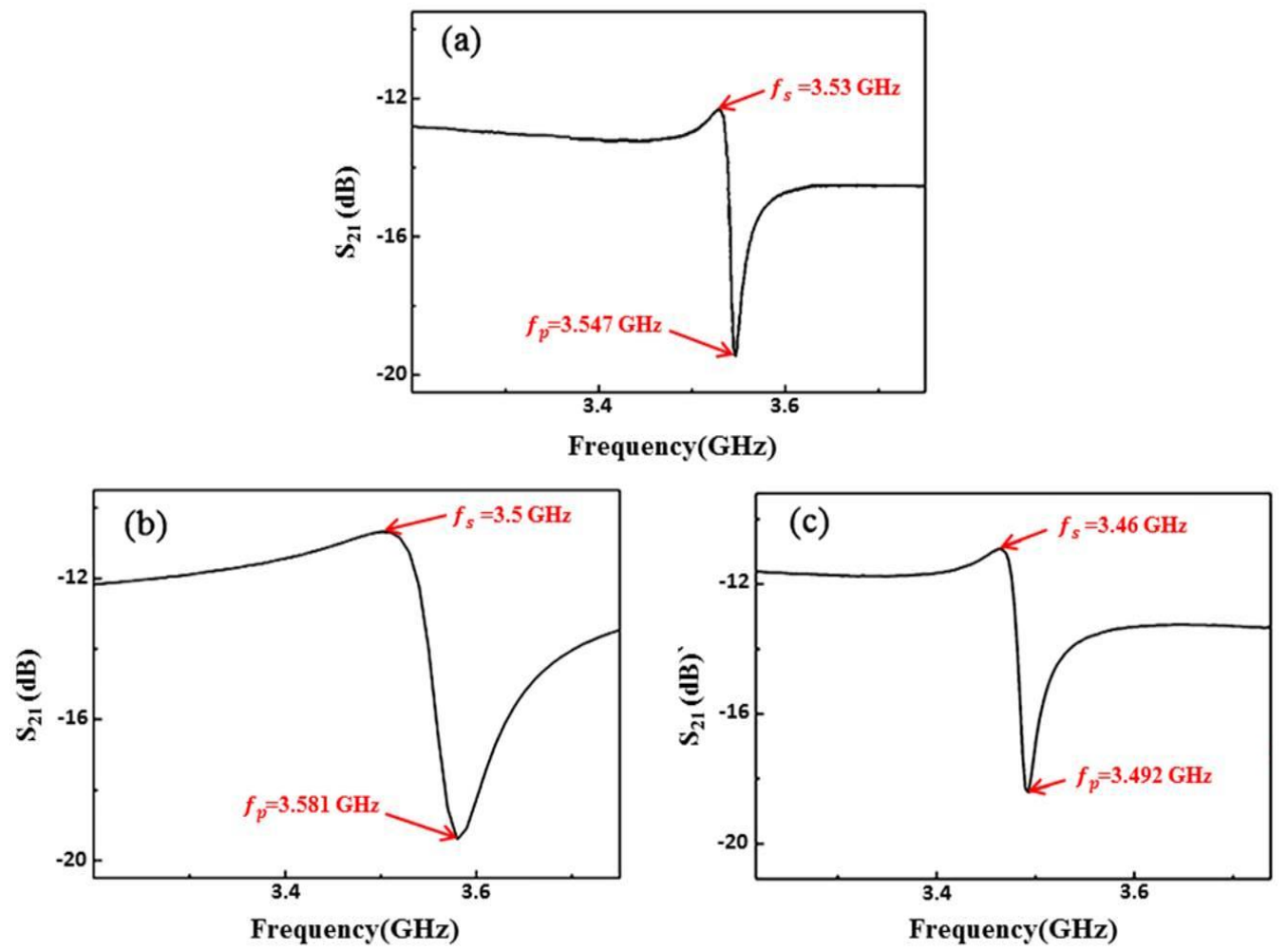

Figure 10. Frequency responses $\left(\mathrm{S}_{21}\right)$ of $\mathrm{SMR}$ resonators using $\mathrm{AlS} \mathrm{CN}$ and $\mathrm{AlN}$ as piezoelectric layers, (a) experimental results of AlN-based resonator, (b) simulation results of AlScN-based resonator, and (c) experimental results of AlScNbased resonator.

In summary, it can be found that in the $3.5 \mathrm{GHz}$ SMR devices made of AlN and AlScN, the insertion loss of the two components is not much different. However, the effective electromechanical coupling coefficient of AlScN-based SMR is almost twice that of AlN-based device. This study successfully used the RF magnetron sputtering system to control the thickness of AlScN film at $731 \mathrm{~nm}$, obtaining the designed $3.5 \mathrm{GHz}$ SMR devices. Figure 12 shows the cross-section of the final fabricated device. The experimental results prove that doping of Sc in the AlN film changes the film's sound velocity and significantly increases its elastic constant, thereby increasing the electromechanical coupling coefficient of the SMR device. 


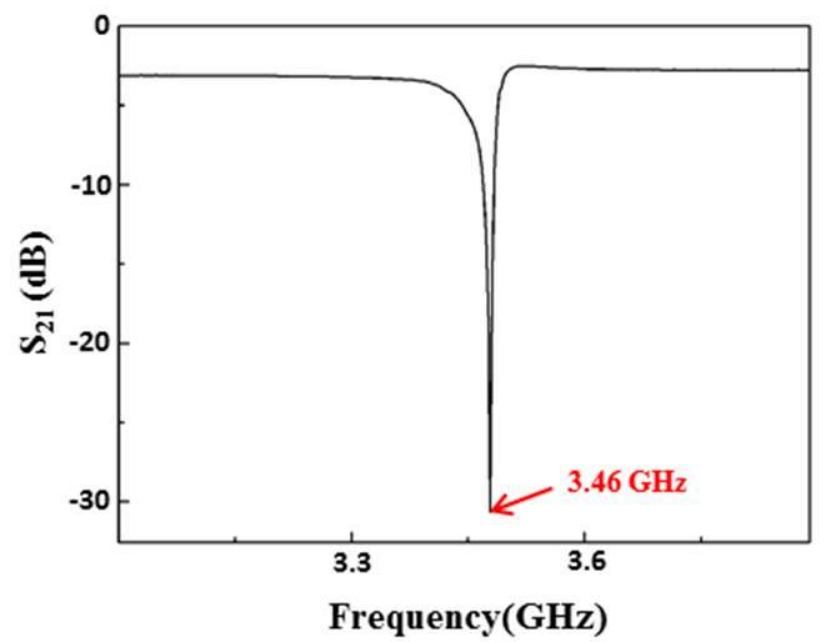

Figure 11. The frequency response $S_{11}$ of the fabricated SMR resonator.

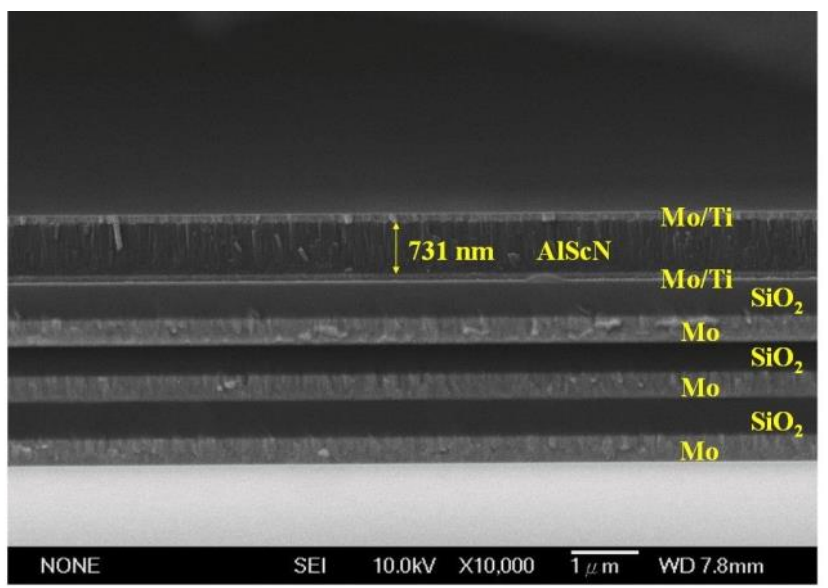

Figure 12. SEM cross-sectional structure diagram of the SMR device.

\section{Conclusions}

High-acoustic-impedance (Mo) and low-acoustic-impedance $\left(\mathrm{SiO}_{2}\right)$ materials were alternately deposited on the silicon substrate through DC and RF magnetron sputtering, respectively, to obtain a high-quality Bragg reflector. An alloy target with atomic ratio of $15 \%$ Sc was adopted in this study. Deposition of a piezoelectric AlScN thin film at a sputtering power of $250 \mathrm{~W}$, a sputtering pressure of 20 mTorr, a nitrogen gas ratio of $20 \%$, and a substrate temperature of $300^{\circ} \mathrm{C}$ resulted in an excellent c-axis (002) crystalline orientation with a dense columnar and pebble-like surface. The XRD diffraction peak angles of the AlScN films are shifted towards lower angles in each crystal phase, compared to those of AlN films. The EDX analysis showed that the percentage of scandium atom in the film is about $4.5 \%$, regardless of the sputtering conditions.

The fabricated resonator exhibited a resonance frequency of $3.46 \mathrm{GHz}$, which was a small deviation from the preset resonance frequency of $3.5 \mathrm{GHz}$. The insertion loss was $-10.92 \mathrm{~dB}$, and the electromechanical coupling coefficient was $2.24 \%$. An AlN piezoelectric film-based device was fabricated and compared with the AlScN-film-based device. According to the obtained results, the electromechanical coupling coefficient of the AlN-based device was $1.17 \%$ and that of the AlScN-based device was $2.24 \%$. The effect of Sc-doped AlScN piezoelectric film was verified. The electromechanical coupling coefficient can be further increased by using alloy targets with a higher atomic ratio of Sc or through co-sputtering technology. 
Author Contributions: Conceptualization, C.-Y.C. and Y.-C.C. (Ying-Chung Chen); methodology, Y.-C.C. (Yu-Cheng Chen); Formal analysis, K.-S.K.; Resources, Y.-C.C. (Yu-Chen Chang); Data curation, C.-Y.C.; Writing-original draft preparation, C.-Y.C.; Writing-review and editing, K.-S.K. and Y.-C.C. (Ying-Chung Chen); Supervision, Y.-C.C. (Ying-Chung Chen). All authors have read and agreed to the published version of the manuscript.

Funding: This study was supported by the Ministry of Science and Technology of the Republic of China, Taiwan. (No. MOST 107-2221-E-110-052-MY2, No. MOST 109-2221-E-110-028 and 110-2622-E110-008) and the Ministry of Education, Taiwan, R.O.C. (107RSI0007).

Institutional Review Board Statement: Not applicable.

Informed Consent Statement: Not applicable.

Data Availability Statement: Not applicable.

Conflicts of Interest: The authors declare no conflict of interest.

\section{References}

1. Ntagwirumugara, E.; Gryba, T.; Zhang, V.Y.; Dogheche, E.; Lefebvre, J.-E. Analysis of frequency response of IDT/ZnO/Si SAW filter using the coupling of modes model. IEEE Trans. Ultrason. Ferroelectr. Freq. Control 2007, 54, 2011-2015. [CrossRef] [PubMed]

2. McCarron, K.; Kline, G.; Martin, J.; Lakin, K. Growth and Characterization of Aluminium Nitride Thin Films for Piezoelectric Devices. In Proceedings of the IEEE Ultrasonics Symposium Proceedings, Chicago, IL, USA, 2-5 October 1988; pp. 673-676.

3. Awang, Z.; Miles, R.; Milne, S.; Tu, Y. Sol-Gel Derived Bulk Acoustic Wave Devices For Cellular Communication Applications. In Proceedings of the IEEE International Conference on Semiconductor Electronics, Penang, Malaysia, 26-28 November 1996; Volume 1, pp. 238-243.

4. Mattila, T.; Oja, A.; Jaakkola, O.; Kiihamäki, J.; Kattelus, H.; Koskenvuori, M.; Rantakari, P.; Tittonen, I.; Seppä, H. Micromechanical Bulk Acoustic Wave Resonator. In Proceedings of the IEEE Ultrasonics Symposium, Munich, Germany, 8-11 October 2002; Volume 1, pp. 945-948.

5. Lanz, R.; Carazzetti, P.; Muralt, P. Surface Micromachined BAW Resonators Based on AlN. In Proceedings of the IEEE Ultrasonics Symposium, Munich, Germany, 8-11 October 2002; Volume 1, pp. 981-983.

6. Hara, M.; Esashi, M. RF MEMS and MEMS Packaging. In Proceedings of the International Symposium on Acoustic Wave Devices for Future Mobile Communication Systems, Chiba, Japan, 3-5 March 2004; pp. 115-122.

7. Kim, H.H.; Ju, B.K.; Lee, Y.H.; Lee, S.H.; Lee, J.K.; Kim, S.W. A noble suspended type thin film resonator (STFR) using the SOI technology. Sens. Actuators A Phys. 2001, 89, 255-258. [CrossRef]

8. Kirby, P.; Potter, M.; Williams, C.; Lim, M. Thin film piezoelectric property considerations for surface acoustic wave and thin film bulk acoustic resonators. J. Eur. Ceram. Soc. 2003, 23, 2689-2692. [CrossRef]

9. Huang, C.-L.; Tay, K.-W.; Wu, L. Fabrication and performance analysis of film bulk acoustic wave resonators. Mater. Lett. 2005, 59, 1012-1016. [CrossRef]

10. Jakkaraju, R.; Henn, G.; Shearer, C.; Harris, M.; Rimmer, N.; Rich, P. Integrated approach to electrode and AlN depositions for bulk acoustic wave (BAW) devices. Microelectron. Eng. 2003, 70, 566-570. [CrossRef]

11. Lee, S.; Lee, J.; Yoon, K.H. Growth of highly c-axis textured AlN films on Mo electrodes for film bulk acoustic wave resonators. J. Vac. Sci. Technol. A Vac. Surf. Film. 2003, 21, 1-5. [CrossRef]

12. Lakin, K.M.; Belsick, J.R.; McDonald, J.P.; McCarron, K.T.; Andrus, C.W. Bulk acoustic wave resonators and filters for 2GHz. In Proceedings of the IEEE MTT-S, Seattle, WA, USA, 3-7 June 2002; pp. 1487-1490.

13. Tay, K.-W.; Huang, C.-L.; Wu, L.; Lin, M.-S. Performance characterization of thin AlN films deposited on Mo electrode for thin-film bulk acoustic-wave resonators. Jpn. J. Appl. Phys. 2004, 43, 5510-5515. [CrossRef]

14. Newell, W. Face-mounted piezoelectric resonators. Proc. IEEE 1965, 53, 575-581. [CrossRef]

15. Lakin, K.; Kline, G.; McCarron, K. Development of miniature filters for wireless applications. IEEE Trans. Microw. Theory Tech. 1995, 43, 2933-2939. [CrossRef]

16. Pinkett, S.; Hunt, W.; Barber, B.; Gammel, P. Broadband Characterization of Zinc Oxide-Based Solidly Mounted Resonators. In Proceedings of the 2002 IEEE International Frequency Control Symposium and PDA Exhibition, New Orleans, LA, USA, 31 May 2002; pp. 15-19.

17. Naik, R.; Lutsky, J.; Reif, R.; Sodini, C.; Becker, A.; Fetter, L.; Huggins, H.; Miller, R.; Pastalan, J.; Rittenhouse, G.; et al. Measurements of the bulk, C-axis electromechanical coupling constant as a function of AlN film quality. IEEE Trans. Ultrason. Ferroelectr. Freq. Control 2000, 47, 292-296. [CrossRef] [PubMed]

18. Lakin, K. Thin film resonator technology. In Proceedings of the IEEE international frequency control sympposium, Tampa, FL, USA, 4-8 May 2005; pp. 765-778.

19. Lanz, R.; Dubois, M.-A.; Muralt, P. Solidly Mounted BAW Filters for the 6 to $8 \mathrm{GHz}$ Range Based on AlN Thin Films. In Proceedings of the 2001 IEEE Ultrasonics Symposium, Atlanta, GA, USA, 7-10 October 2001; Volume 1, pp. 843-846. 
20. Loebl, H.; Metzmacher, C.; Peligrad, D.; Mauczok, R.; Klee, M.; Brand, W.; Milsorn, R.; Lok, P.; Van Straten, F.; Tuinhout, A.; et al. Solidly mounted bulk acoustic wave filters for the GHz frequency range. In Proceedings of the 2002 IEEE Ultrasonics Symposium, Munich, Germany, 8-11 October 2002; Volume 1, pp. 919-923.

21. Kim, S.; Kim, J.; Park, H.; Yoon, G. AlN-based film bulk acoustic resonator devices with W/SiO multilayers reflector for rf bandpass filter application. J. Vac. Sci. Technol. B Microelectron. Nanometer Struct. 2001, 19, 1164-1169. [CrossRef]

22. Dubois, M.-A.; Muralt, P.; Matsumoto, H.; Plessky, V. Solidly Mounted Resonator Based on Aluminum Nitride Thin Film. In Proceedings of the 1998 IEEE Ultrasonics Symposium, Sendai, Japan, 5-8 October 1998; Volume 1, pp. $909-912$.

23. Lee, S.-H.; Yoon, K.H.; Lee, J.-K. Influence of electrode configurations on the quality factor and piezoelectric coupling constant of solidly mounted bulk acoustic wave resonators. J. Appl. Phys. 2002, 92, 4062-4069. [CrossRef]

24. Tasnádi, F.; Alling, B.; Höglund, C.; Wingqvist, G.; Birch, J.; Hultman, L.; Abrikosov, I.A. Origin of the anomalous piezoelectric response in wurtzite ScxAl1-xN alloys. Phys. Rev. Lett. 2010, 104, 137601-137604. [CrossRef] [PubMed]

25. Farrer, N.; Bellaiche, L. Properties of hexagonal ScN versus wurtzite GaN and InN. Phys. Rev. B 2002, 66, 201203. [CrossRef]

26. Alsaad, A.; Ahmad, A. Piezoelectricity of ordered $\left(\mathrm{Sc}_{x} \mathrm{Ga} 1-_{x} \mathrm{~N}\right)$ alloys from first principles. Eur. Phys. J. B 2006, 54, 151-156. [CrossRef]

27. Turner, R.; Fuierer, P.; Newnham, R.; Shrout, T. Materials for high temperature acoustic and vibration sensors: A review. Appl. Acoust. 1994, 41, 299-3244. [CrossRef]

28. Kim, S.-H.; Kim, J.-H.; Lee, J.-K.; Lee, S.-H.; Yoon, K.H.; Sang-Hee, K.; Jong-Heon, K.; Jeon-Kook, L.; Si-Hyung, L.; Hyun, Y.K. Bragg Reflector Thin Film Resonator Using Aluminium Nitride Deposited by RF Sputtering. In Proceedings of the Asia-Pacific Microwave Conference, Sydney, NSW, Australia, 3-6 December 2000; pp. 1535-11538.

29. Loebl, H.; Metzmacher, C.; Milsom, R.; Lok, P.; Van Straten, F.; Tuinhout, A. RF bulk acoustic wave resonators and filters. J. Electroceramics 2004, 12, 109-118. [CrossRef]

30. Larson, J.; Bradley, P.; Wartenberg, S.; Ruby, R. Modified butterworth-van dyke circuit for FBAR resonators and automated measurement system. In Proceedings of the 2000 IEEE Ultrasonics Symposium. Proceedings. An International Symposium (Cat. No.00CH37121), San Juan, PR, USA, 22-25 October 2000; Volume 1, pp. 863-868.

31. Lakin, K.M. Thin film resonators and filters. In Proceedings of the 1999 IEEE Ultrasonics Symposium. Proceedings. International Symposium (Cat. No. 99CH37027), Tahoe, NV, USA, 17-20 October 1999; Volume 2, pp. 895-906.

32. Akiyama, M.; Kamohara, T.; Kano, K.; Teshigahara, A.; Takeuchi, Y.; Kawahara, N. Enhancement of piezoelectric response in scandium aluminum nitride alloy thin films prepared by dual reactive cosputtering. Adv. Mater. 2009, 21, 593-596. [CrossRef] [PubMed]

33. Wang, W.; Mayrhofer, P.M.; He, X.; Gillinger, M.; Ye, Z.; Wang, X.; Bittner, A.; Schmid, U.; Luo, J. High performance AlScN thin film-based surface acoustic wave devices with large electromechanical coupling coefficient. Appl. Phys. Lett. 2014, 105, 133502. [CrossRef] 